\title{
Steroid Hormones as Transporters to Carry Exogenous Macromolecules into the Target Cell Nuclei in Vivo
}

\section{Toshikazu Nishimura}

Aichi Medical University School of Medicine, Yazako, Nagakute, Aichi 480-1195, Japan

\section{Email address:}

nishimur@aichi-med-u.ac.jp (T. Nishimura)

\section{To cite this article:}

Toshikazu Nishimura. Steroid Hormones as Transporters to Carry Exogenous Macromolecules into the Target Cell Nuclei in Vivo. American Journal of Life Sciences. Special Issue: Biology and Medicine of Peptide and Steroid Hormones. Vol. 3, No. 3-2, 2015, pp. 53-57. doi: 10.11648/j.ajls.s.2015030302.20

\begin{abstract}
Upon injection into the vascular system of rats, testosterone-bovine serum albumin conjugate (testosterone-BSA) is taken up by cells via the process of endocytosis. When it is taken up by the target cells of testosterone such as spermatogenic cells, it enters the nuclei of the cells. However, testosterone-BSA does not enter the nuclei of the non-target cells such as hepatocytes and thymocytes. Similarly, hydrocortisone-BSA conjugate enters the nuclei of its target cells such as hepatocytes and thymocytes. In the vesicular trafficking of testosterone-BSAs into the nucleoplasm, the vesicle membrane is likely to fuse with a nuclear hemifusion diaphragm. IgG coupled with hydrocortisone also enters the hormone-target cell nuclei, with its antigenicity kept intact. These results suggest that steroid hormones could act as transporters for conveying exogenous macromolecules into the target cell nuclei in vivo. Our studies provide a novel insight to the functions of steroid hormones.
\end{abstract}

Keywords: Steroid-Protein Conjugates, Vesicular Trafficking, Nuclear Diaphragm, Target Cell Nuclei, Intact Antigenicity, In Vivo

\section{Introduction}

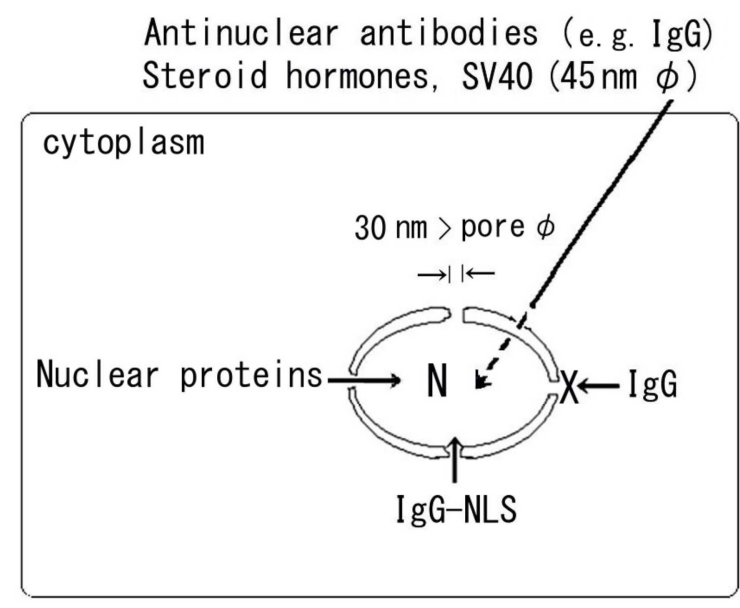

Fig. 1. A model of nuclear transport. SV40, steroid hormones and IgG enter the nucleus (N) from the outside of the cell. Nuclear proteins in the cytoplasm enter the nucleus through NPCs, but IgG cannot enter the nucleus from the cytoplasm. IgG conjugated with synthetic peptides containing nuclear localization signal sequence (NLS) are able to enter the nucleus from the cytoplasm.

Steroid hormones can circulate in blood plasma in three different forms: albumin-bound, steroid hormone-binding globulin-bound, and free $[1,2]$. In the classical genomic model of steroid hormone action, free lipophilic hormones cross the cell membrane under passive transport, bind to their intracellular receptors in the target cells to form hormone-receptor complexes and subsequently move to the nucleus to exert their genomic effects [3] (Fig. 1). However, there are also reports on cell membrane-initiated effects, or non-genomic activities of steroid hormones [4, 5]. Such reports indicate that steroid hormones might have some functions yet unknown, such as the transportation of various macromolecules into the target cell nuclei.

\section{A Hypothesis on the Evolution of Life Based on Genetics}

The purpose of my research was explaining the evolution of life based on genetics. The concept of heredity is based on the knowledge that all genes in the progenies are inherited from the parents. In the evolution of life, however, progenies tend to develop some variations in genes which do not originate from their parents. In fact, a great part of the evolution is the results of genetic changes occurring inside of the cells. Viruses are 
one of the major contributors to such genetic changes. Papovavirus virions such as simian virus 40 (SV40) are composed of proteins and DNA, and are able to enter the nucleus from the exterior of the cell [6] (Fig. 1). When SV40 infects 3T3 cells (mouse embryonic fibroblast), the virus induces cell transformation. This phenomenon likely portrays an example of the evolution in the unicellular organisms. Therefore, I hypothesized that the evolution may have been caused by an accumulation of exogenous genes in a cell nucleus.

\section{Vesicular Transport of SV40 into the Nucleus}

In some infection processes, virus-containing vesicles fuse with the outer nuclear membrane, delivering the virus particles into the perinuclear cisterna [7, 8]. Single-bilayer diaphragms, such as shared bilayers, have been observed in the processes of fusion between the cell membrane and the secretory vacuole membrane [9], and between the cell membranes of two myoblasts [10], under transmission electron microscope. Membrane fusion without cytoplasmic fusion, referred to as a shared bilayer or hemifusion, can occur between two membranes [11]. In the search for other entryways to the nucleus, migration of SV40 was pursued in cultured cells, using ferritin and concanavalin A as cell membrane markers. Ferritin particles introduced into the cytoplasm did not enter the nucleus. In contrast, SV40-containing vesicles with ferritin particles were observed close to a single-bilayer nuclear membrane, or a hemifusion diaphragm [12]. The nucleoplasmic side of the hemifusion diaphragm was covered with electron-dense materials, and cell membrane markers were localized along the nucleoplasmic side of the inner nuclear membrane (Fig. 2). These results suggest that SV40-containing vesicle membrane fuse to hemifusion diaphragms in the nuclear envelope in order to transport virus particles into the nucleoplasm, and that the exogenous macromolecules used here as cell membrane markers were transported into the nucleus in this manner [12].

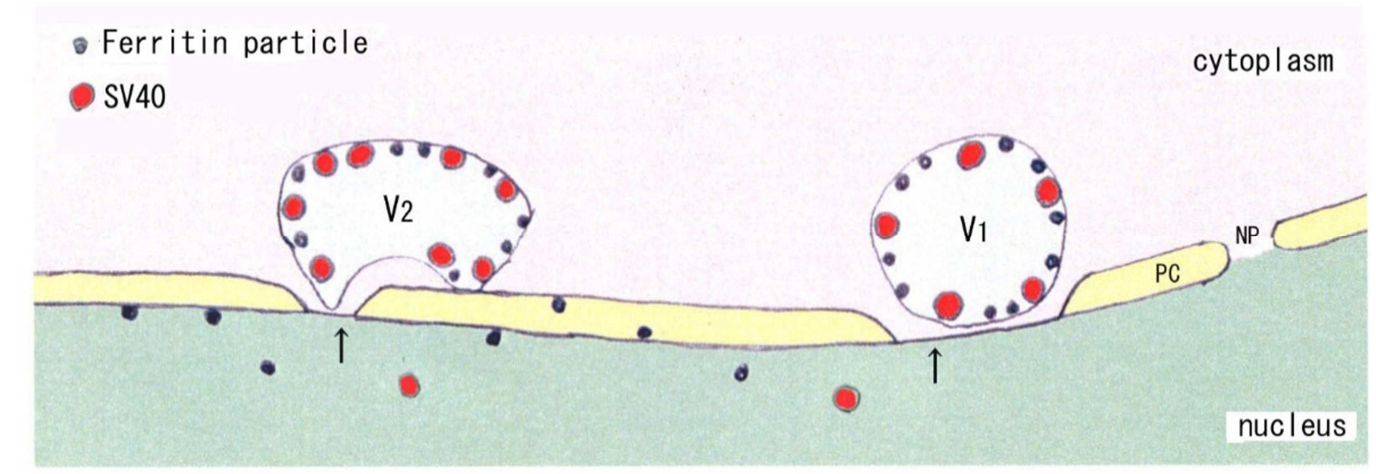

Fig. 2. A representation of the nuclear transfer process of endocytosed SV40. Vacuoles (V1, V2) containing ferritin particles and SV40 are located near the single membrane-like envelopes, or diaphragms (arrows). At those areas, the perinuclear cisterna (PC) is not identified. A part of vacuole membrane (V2) is in contact with the outer nuclear membrane. SV40 is found in the nucleus. Several ferritin particles are located in the perinuclear cisterna, and further in the nucleoplasm near the vacuoles. The particles are not identified within the nuclear pores (NP).

\section{A Hypothesis about Antinuclear Antibodies}

In addition to SV40, it is well-know that antinuclear antibodies such as immunoglobulin $\mathrm{G}(\mathrm{IgG})$ can also enter the nucleus from the exterior of a cell (Fig. 1). Substances such as SV40 tumor antigen and nuclear proteins migrate into the nucleus from the cytoplasm [13, 14]. IgG with synthetic peptides containing nuclear localization signal sequence such as that of SV40 tumor antigen moves into the nucleus from the cytoplasm [15] (Fig. 1) by active transport through nuclear pore complexes (NPCs). Macromolecules such as native IgG which do not possess nuclear localization signals are otherwise not actively transported by NPCs, and are not likely to pass freely the cell or nuclear membrane by passive transport, either. Accordingly, the occurrence of autoimmune diseases implies that there must be other routes which allow the IgG nuclear entry besides the fusion of the outer nuclear membrane and vesicle's membrane. In addition, mechanisms that disturb the digestion by lysosomal enzymes must also exist, which protect the IgG during its vesicular transport from the outside of the cell to the nucleus.

Already back in the early 1970 s, we (my team back then in Osaka City University and Osaka University, Japan) have developed a method to introduce exogenous substances into the cytoplasm of cultured cells, using inactivated Sendai virus [16]. Another possible method is to make use of the antinuclear antibodies. Immunoglobulin $\mathrm{G}$ has been identified as an antinuclear antibody in some autoimmune diseases, targeting endogenous contents within the cell nucleus. In order to verify whether $\operatorname{IgG}$ moves into the nucleus from the cytoplasm, IgG was introduced to the cytoplasm [17, 18]. However, IgG did not enter the nucleus, as shown in Figure 1. Out of the possible combinations with the substances which can enter the nucleus from the exterior of the cell (Fig. 1), it was speculated that coupling with steroid hormones may enable the nuclear transfer of IgG. For example 
hydrocortisone has specific stimulatory effects on the epithelial cells of cultured rat prostate explants [19], even though they don't express glucocorticoid receptor. This fact strongly suggests another effects of steroid hormone.

\section{Steroid Hormones as Transporters for Carrying Exogenous Macromolecules into the Target Cell Nuclei in Vivo}

Steroid hormones conjugated with bovine serum albumin (steroid-BSAs) are used for the analyses of the binding sites of steroid hormones to cell membranes [20, 21]. Pietras and Szego suggested that endocytotic vesicles appear to serve as vehicles for nuclear migration of steroid hormones [22]. Steroid hormone-binding globulin coupled with $\left[{ }^{3} \mathrm{H}\right]$-testosterone is internalized by receptor-mediated endocytosis in spermatogenic cells, which are target cells of testosterone, and then enters the nuclei of these cells in vitro $[23,24]$. Our group showed that colloidal gold embedded in epoxy resin becomes visible as silver deposits on the sections after silver enhancement [25]. The gold particles seem to be stable in the lysosome. Upon injection into the vascular system of rats, testosterone-bovine serum albumin conjugate labeled with $2 \mathrm{~nm}$ colloidal gold (testosterone-BSA-gold) is taken up by endocytosis into the target cells of testosterones such as round spermatids, and then enters the nucleoplasm [25, 26]. In contrast, the nuclei of cells which are not targeted by testosterone such as thymocytes and hepatocytes showed very few silver deposits implying the presence of testosterone-BSA-gold [25]. These results suggest that the nuclear entry of testosterone-BSA-gold is specific to the target cells of testosterone; in other words, testosterone-BSA-gold does not enter the non-target cell nuclei. From the distribution of silver deposits, it has become clear that hydrocortisone-BSA-gold conjugates injected into rats enter the target cell nuclei such as hepatocytes and thymocytes. Together with the aforementioned studies on testosterone-BSA-gold conjugates, it indicates that the fate of gold labeled-steroid-BSAs may be decided at the cell membrane level [27].

In order to clearly show the migration route of testosterone-BSA-gold to the nucleoplasm through the nuclear envelope by vesicular trafficking, the round spermatids were observed under electron microscope. In spermiogenesis, the nuclear envelope of a round spermatid is divided into two forms, as a consequence of acrosome expansion over the anterior pole of the nucleus: 1) in the post-acrosomal region of the nuclear envelope, the nuclear pores continue to be present during the expansion of acrosome. 2) in the subacrosomal region, the two nuclear membranes are in close apposition and devoid of pores [28, 29]. In round spermatids of the rats injected with testosterone-BSA-gold, the silver deposits were present on the cell membrane, vesicles, Golgi region, acrosome, subacrosomal space, both the post-acrosomal and the subacrosomal nuclear envelope, and the nucleoplasm. The silver deposits were also found in the perinuclear cisterna of post-acrosomal nuclear envelope, but not in the nuclear pore [26] (Fig. 3). In an observation of the post-acrosomal nuclear envelope without silver enhancement, the outer nuclear membrane showed many irregular invaginations toward the inner nuclear membrane. Furthermore, a double-membrane-like vesicle seemed to be present in the nuclear envelope. A vesicle containing gold particles was present in the pit formed by the invagination of the outer nuclear membrane (Fig. 3). These results suggest that testosterone-BSA-gold, being a macromolecule, is transported by the vesicles from the outside of the cells to the nucleoplasm [26]. This route resembles the entryway proposed for nuclear migration of SV40 in 1991 [12].

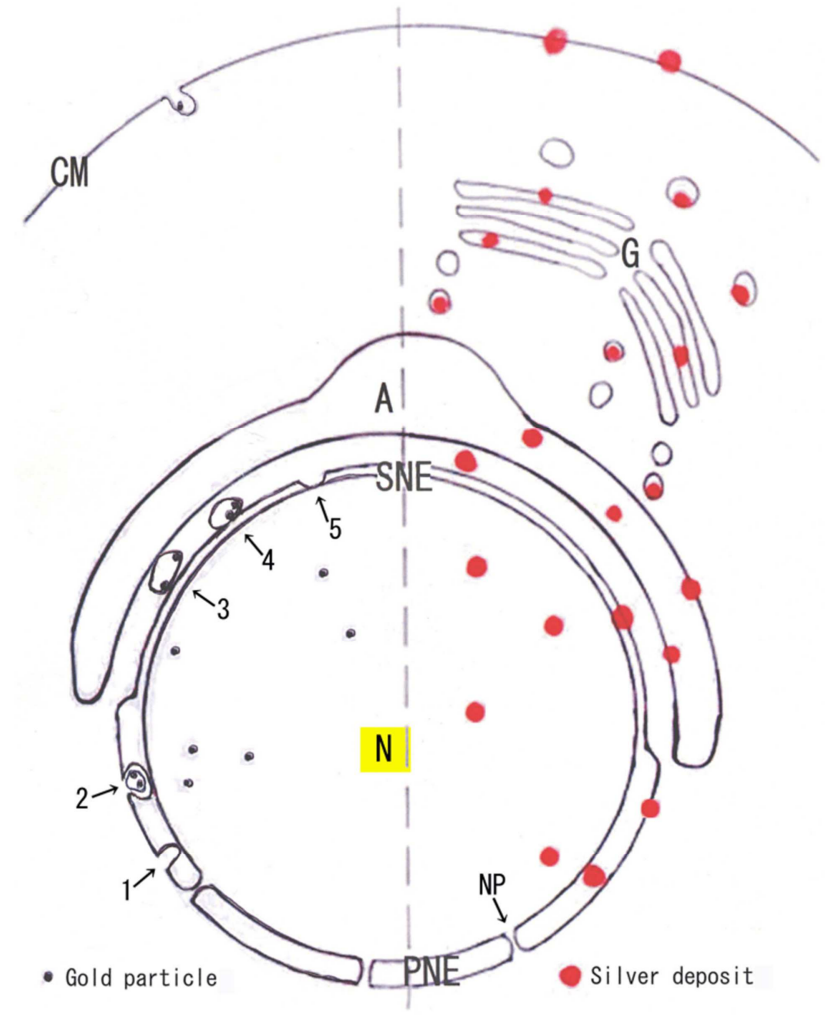

(L) Without silver enhancement $\quad$ (R) After silver enhancement

Fig. 3. Representation of the distribution of $2 \mathrm{~nm}$ gold particles (silver deposits), implying the location of testosterone-BSA-gold in the round spermatids of rats. Right half $(R)$ represents the image of cells after silver enhancement. The silver deposits are present on the cell membrane (CM), vesicles, Golgi region $(G)$, acrosome $(A)$, subacrosomal space, both the post-acrosomal-and the subacrosomal nuclear envelope (PNE and SNE, respectively) and the nucleoplasm (N), but not in the nuclear pore (NP). A deposit is present in the perinuclear cisterna of post-acrosomal nuclear envelope. Left half $(L)$ represents the cell images without silver enhancement. The outer membrane of the post-acrosomal nuclear envelope shows many irregular invaginations toward the inner nuclear membrane (arrow 1). A double-membrane-like vesicle is present in the nuclear envelope (arrow 2). In the subacrosomal space, vesicles containing gold particles are found close to the inner membrane of acrosome (arrow 3), or in contact with the SNE (arrow 4). A part of SNE is manifesting a single-bilayer diaphragm (arrow 5).

From the distribution of the silver deposits in the subacrosomal region, we suggested that testosterone-BSA-gold is also transported from the acrosome to the nucleoplasm through the subacrosomal nuclear 
envelope (SNE), which is devoid of pores [26]. In the observation without silver enhancement, some vesicles containing gold particles were located close to the inner membrane of acrosome in subacrosomal space, or were found to be in contact with the SNE, of which the nucleoplasmic side was covered with electron-dense material. Furthermore, there were the diaphragms, that is, single-bilayer nuclear membranes in SNE, which seemed to partially lack the nuclear lamina (Fig. 3). These results indicate the possibility that the membranes of vesicles fuse with shared bilayers in the SNE [30].

Then, we immunocytochemically investigated whether BSA in the steroid-BSAs remains intact in the hormone-target cell nuclei. For this purpose, testosterone-BSA, hydrocortisone-BSA or corticosterone-BSA was injected into the rats. BSA conjugated with steroid hormones could enter the hormone-target cell nuclei while maintaining its antigenicity. These results suggest the possibility that steroid hormone can transport the macromolecule to nucleus [31]. $\mathrm{IgG}$ antibodies introduced into the cells are functionally stable in the cytoplasm. When an antibody is introduced in the cytoplasm, the antibody reacts to its antigen [32]. Bovine IgG coupled with hydrocortisone injected into rat vascular system enters the hormone-target cell nuclei in the liver, maintaining the antigenicity [33]. This last finding also confirms that steroid hormones act as carriers to convey exogenous proteins into the target cell nuclei. Then, it may be possible that foreign DNA can be transported to the nucleus of target cells, such as spermatogenic cells, by associating with some protein-steroid hormone complexes, and they contribute to causing the evolution. However, whether it is more efficient to conjugate DNA with carrier proteins or steroid hormone in sending it into spermatogenic cell nuclei is still unknown.

\section{Conclusion}

Steroid hormones can function as transporters for carrying exogenous macromolecules into the target cell nuclei in vivo. The fate of proteins coupled with steroid hormone seems to be decided at the cell membrane level.

\section{References}

[1] Horton RJ. Testicular steroid transport, metabolism, and effects In Principles and Practice of Endocrinology and Metabolism (K.L. Becker, J.P. Bilezikian, D.L. Loriaux, W.J. Bremner, RW Rebar, W Hung, GL Robertson, CR Kahn and L Wartofsky, eds.) J B Lippincott Company, Philadelphia, 1990, pp937-942.

[2] Pardridge WM. Selective delivery of sex steroid hormones to tissues in vivo by albumin and by sex hormone-binding globulin. Ann. NY Acad Sci 1988; 538: 173-192.

[3] Jensen EV and DeSombre ER. Mechanism of action of the female sex hormones. Ann Rev Biochem, 1972; 41: 203- 230.

[4] Revelli A, Massobrio M, Tesarik J. Nongenomic actions of steroid hormones in reproductive tissues. Endocine Rev 1998; 19: 3-17
[5] Wehling M. Specific, nongenomic actions of steroid hormones. Annu Rev Physiol 1997; 59: 365-393

[6] Hummeler K, Tomassini N, Sokol F. Morphological aspects of the uptake of simian virus 40 by permissive cells. J Virol 1970; 6: 87-93.

[7] Maul GG, Rovera G, Vorbrodt A, Abramczuk J. Membrane fusion as a mechanism of simian virus 40 entry into different cellular compartments. J Virol 1978; 28: 936-944.

[8] Nishimura T, Kawai N, Kawai M, Notake K, Ichihara I. Fusion of SV40-induced endocytotic vacuoles with the nuclear membrane. Cell Struct Funct 1986; 11: 135-141.

[9] Pinto da Silva P, Nogueira ML. Membrane fusion during secretion. a hypothesis based on electron microscope observation of Phytophthora Palmivora zoospores during encystment. J Cell Biol 1977; 73: 161-181.

[10] Kalderon N, Gilula NB. Membrane events involved in myoblast fusion. J Cell Biol 1979; 81: 411-425.

[11] Chernomordik LV, Kozlov MM. Membrane hemifusion: crossing a chasm in two leaps. Cell 2005; 123: 375-382.

[12] Nishimura T, Kawai N, Ichihara I. Interaction of endocytotic vacuoles with the inner nuclear membrane in simian virus 40 entry into CV-1 cell nucleus. Cell Struct Funct 1991; 16: 441-445.

[13] Lanford RE, Kanda P, Kennedy RC. Induction of nuclear transport with a synthetic peptide homologous to the SV40 T antigen transport signal. Cell 1986; 46; 575-82.

[14] Yamaizumi M, Uchida T, Okada Y, Furusawa M, Mitsui H. Rapid transfer of non-histone chromosomal proteins to the nucleus of living cells. Nature 1978; 273: 782-4.

[15] Yoneda Y, Hieda M, Nagoshi E, Miyamoto Y. Nucleocytoplasmic protein transport and recycling of Ran. Cell Struct Funct 1999; 24: 425-433.

[16] Furusawa M, Nishimura T, Yamaizumi M, Okada Y. Injection of exogenous substances into single cells by cell fusion. Nature 1974; 249: 449-50.

[17] Nishimura T, Furusawa M, Yamaizumi M, Okada Y. Method for intracellular injection by cell fusion using erythrocyte ghosts. Cell Struct Funct 1976; 1: 197-200.

[18] Furusawa M, Yamaizumi M, Nishimura T, Uchida T, Okada Y. Use of erythrocyte ghosts for injection of substances into animal cells by cell fusion. Methods Cell Biol 1976; 14: 73-80.

[19] Ichihara I, Santti RS, Pelliniemi LJ. Effects of testosterone, hydrocortisone and insulin on the fine structure of the epithelium of rat ventral prostate in organ culture. Z Zellforsch Mikrosk Anat 1973; 143: 425-38.

[20] Ramirez VD, Zheng J. Membrane sex-steroid receptors in the brain. Front Neuroendocrinol 1996; 17: 402-439.

[21] Rao BR, Patrick TB, Sweet F. Steroid-albumin conjugate interaction with steroid-binding proteins. Endocrinology 1980; 106: 356-362.

[22] Pietras RJ, Szego CM. Specific internalization of estrogen and binding to nuclear matrix in isolated uterine cells. Biochem Biophys Res Commun 1984; 123: 84-91. 
[23] Gerard A, En Nya A, Egloff M, Domingo M, Degrelle H, and Gerard H. Endocytosis of human sex steroid-binding protein in monkey germ cells. Ann NY Acad Sci. 1991; 637: 258-276.

[24] Gerard H, Gerard A, En Nya A, Felden F, and Gueant JL. Spermatogenic cells do internalize Sertoli androgen-binding protein: A transmission electron microscopy autoradiographic study in the rat. Endocrinology 1994; 134: 1515- 1527.

[25] Nishimura T, Ichihara I. Nuclear concentration of gold labeled-testosterone-bovine serum albumin conjugate injected intravenously in the hormone-target cells of rat. Cell Struct Funct 1997; 22: 433-442.

[26] Nishimura T, Nakano T. Nuclear translocation of gold labeled-testosterone-bovine serum albumin conjugate through the nuclear double membranes in rat spermatids. Cell Struct Funct 1997; 22: 621-629.

[27] Nishimura T, Nakano T. Nuclear localization of gold labeled-hydrocortisone-bovine serum albumin conjugate injected intravenously into the hormone-target cells of rat. Cell Struct Funct 1999; 24: 227-35.

[28] Dym M. The male reproductive system. In: Weiss L (ed) Cell and Tissue Biology. A Textbook of Histology, Urban \& Schwarzenberg Inc., Baltimore Munich, 1988, pp 929-972
[29] Fawcett DW, Raviola E. Male reproductive system. In: Bloom and Fawcett, a textbook of histology, 12th edn. Chapman \& Hall, New York, 1994, pp 768-815

[30] Nishimura T, Nakano T. Vesicles in the subacrosomal space and partial diaphragms in the subacrosomal nuclear envelope of round spermatids of a rat injected intravenously with gold labeled-testosterone-bovine serum albumin conjugate: vesicular trafficking from acrosome to nucleus. Okajimas Folia Anat Jpn 2002; 79: 15-23.

[31] Nishimura T, Nakano T. Immunocytochemical localization of bovine serum albumin (BSA) in the liver and testis of rats injected with testosterone-BSA, hydrocortisone-BSA or corticosterone-BSA. Cell Struct Funct 2000; 25: 161-169.

[32] Yamaizumi M, Uchida T, Takamatsu K, Okada Y. Intracellular stability of diphtheria toxin fragment A in the presence and absence of anti-fragment A antibody. Proc Natl Acad Sci USA 1982; 79: 461-5.

[33] Nishimura T, Nakano T. Nuclear localization of bovine immunoglobulin $\mathrm{G}(\mathrm{bIgG})$ in the liver of rats injected with hydrocortisone-bIgG conjugate intravenously. Okajimas Folia Anat Jpn 2001; 78: 107-114. 